\title{
Characterization by phenotypic and genotypic methods of metallo- $\beta$-lactamase-producing Pseudomonas aeruginosa isolated from patients with cystic fibrosis
}

\author{
YONGWEI LI ${ }^{1,2}$, XIAOQIAN ZHANG ${ }^{2}$, CHUNXIA WANG $^{2}$, YUE HU $^{2}$, \\ XIAOBIN NIU ${ }^{2}$, DONGXU PEI ${ }^{2}$, ZHIQIANG $\mathrm{HE}^{2}$ and YONGYI BI ${ }^{1}$ \\ ${ }^{1}$ Wuhan University School of Public Health, Wuhan, Hubei 430071; ${ }^{2}$ Department of Clinical Laboratory, \\ Henan Hospital of Traditional Chinese Medicine, Zhengzhou, Henan 450002, P.R. China
}

Received November 29, 2013; Accepted June 5, 2014

DOI: $10.3892 / \mathrm{mmr} .2014 .2685$

\begin{abstract}
Pseudomonas aeruginosa continues to be a predominant cause of infections with high intrinsic resistance to antibiotics, resulting in treatment failure. P. aeruginosa is the leading cause of respiratory infections among cystic fibrosis $(\mathrm{CF})$ patients. Resistance to carbapenem antibiotics among $P$. aeruginosa has been reported. Thus, this study was undertaken to characterize the metallo- $\beta$-lactamase (MBL) production of $P$. aeruginosa by phenotypic and genotypic methods. A total of 572 sputum samples were collected from cystic fibrosis patients along with the patient demographic details in a questionnaire. In total, 217 P. aeruginosa isolates were collected and an antibiogram revealed that $159(73.3 \%)$ and $141(64.9 \%)$ of these colonies exhibited resistance to imipenem and meropenem, respectively. Ceftazidime and tobramycin resistance were both identified in $112(51.6 \%)$ isolates, and resistance to piperacillin-tazobactam, gatifloxacin and netilmicin was detected in 96 (44.2\%) respective samples. A total of $62(28.6 \%)$ respective samples were resistant to cefoperazone, cefepime and ceftriaxone. The least antibiotic resistance was shown to amikacin and ceftizoxime with $51(23.5 \%)$ and $32(14.7 \%)$ respective colonies resistant to the antibiotics. The minimum inhibitory concentration (MIC) for imipenem revealed a reduction in the MIC values. MBL screening by the zone enhancement method using ceftazidime plus EDTA discs demonstrated that $63(56.25 \%)$ of the colonies were positive for MBL. A total of $53(84.1 \%)$ samples expressed blaVIM and 48 (76.1\%) expressed blaIMP genes, as detected by duplex polymerase chain reaction. In conclusion, carbapenem resistance is of great clinical concern in cystic fibrosis patients with $P$. aeruginosa infection. Therefore, mandatory regular
\end{abstract}

Correspondence to: Dr Yongwei Li, Department of Clinical Laboratory, Henan Hospital of Traditional Chinese Medicine, 6 Donfeng Road, Zhengzhou, Henan 450002, P.R. China

E-mail: yongwei73@gmail.com

Key words: Pseudomonas aeruginosa, metallo- $\beta$-lactamase, cystic fibrosis screening and monitoring the resistance in P.aeruginosa among $\mathrm{CF}$ patients is required.

\section{Introduction}

The fundamental aspect of interactions among microbes and the host organism is the ability of the pathogen to entrench itself and establish a persistent infection. Cystic fibrosis (CF) is an autosomal recessive genetic disease (6); the opportunistic pathogen Pseudomonas aeruginosa may cause chronic lung disease in CF patients, depending on the genetic adaptation of the pathogen, and is a prevalent pathogen in CF patients with pulmonary infection (1-5). Worldwide, $80 \%$ of CF patients were found to be infected with $P$. aeruginosa $(7,8)$. Pseudomonas colonization in the lungs of $\mathrm{CF}$ patients results in tissue destruction and reduced respiratory function (9). Identification of $\mathrm{CF}$ isolates is difficult due to the phenotypic diversity, including the formation of mucoid colonies, loss of pigment and synthesis of rough lipopolysaccharides (10). However, identification using genotypic methods may evade this problem of identifying the variable phenotypes.

A high level of resistance has been exhibited by $P$. aeruginosa to numerous antimicrobials. Active efflux pump systems are of great importance in $P$. aeruginosa resistance. The MexA-mexB-oprM operon significantly contributes to the increased resistance of opportunistic pathogens (11). The rapid increase in antimicrobial resistance among Pseudomonas spp has resulted in extensive investigations aimed at understanding the factors that promote the emergence of antimicrobial resistance in Pseudomonas. Since Pseudomonas is inherently resistant to a number of antibiotics, infection with this bacterium is a serious problem in the treatment of CF patients. Carbapenem antibiotics, including imipenem and meropenem, are used in the treatment of infections caused by $P$. aeruginosa (12); however, carbapenem resistance among $P$. aeruginosa strains has been reported in recent years (13). The production of $\beta$-lactamase and metallo- $\beta$-lactamase (MBL), and reduced penetration of the drugs are challenging factors in the therapeutic management of these infections $(13,14)$.

The MBLs are classified into three subgroups: B1, B2 and B3, according to their molecular structure. GIM, VIM, 
SPM and IMP are genes in integrons, which integrate into chromosomes or plasmids $(15,16)$. The genes involved in MBL production are either plasmid- or chromosome-mediated, and are transferred horizontally. This horizontal transfer of genes poses a health threat through spreading of resistance among other Gram-negative bacteria (17). Knowledge of these enzymes conferring resistance is required to prevent the spread of the infection among clinical samples. Numerous nosocomial outbreaks of $P$. aeruginosa producing metallo-lactamases have been reported, with an urgent requirement to implement infection control programs (18-21). Thus, the present study was undertaken to detect the presence of MBL-producing P. aeruginosa isolates from the sputa of CF patients by phenotypic and genotypic methods.

\section{Materials and methods}

Bacterial strains. A total of $572 \mathrm{CF}$ patients were included in the study from Henan Hospital of Traditional Chinese Medicine (Zhengzhou, China). Sputum samples from patients with $\mathrm{CF}$ were collected and processed according to standard methods (Clinical and Laboratory Standards Institute ${ }^{\circledR}$; CLSI Guidelines, 2012). All demographic details, including age, gender and history of antibiotic usage were collected. P. aeruginosa was isolated and characterized using biochemical methods. The present study was approved by the ethical committee of Henan Hospital of Traditional Chinese Medicine. Written informed consent was obrained from the patient's families.

Antimicrobial susceptibility analysis. The following antibiotics (Oxoid Ltd., Basingstoke, UK) were used for the antimicrobial susceptibility analysis by Kirby Bauer's disc diffusion method according to CLSI guidelines (23): Amikacin (30 mg), gentamicin (10 mg), netilmicin (30 mg), tobramycin (10 mg), cefoperazone $(75 \mathrm{mg})$, cefepime $(30 \mathrm{mg})$, ceftazidime $(30 \mathrm{mg})$, ceftriaxone (30 mg), ceftizoxime (30 mg), ciprofloxacin $(5 \mathrm{mg})$, gatifloxacin $(5 \mathrm{mg})$, imipenem $(10 \mathrm{mg})$, meropenem $(10 \mathrm{mg})$ and piperacillin-tazobactam $(100 / 10 \mathrm{mg})$. P. aeruginosa ATCC 27853, obtained from the Microbology Laboratory at Henan Hospital of Traditional Chinese Medicine, served as a control.

Minimum inhibitory concentration (MIC). The MIC was determined on imipenem-resistant isolates using the agar dilution method, with serial dilution of the imipenem powder at a concentration range of $0.06-512 \mu \mathrm{g} / \mathrm{ml}$. A volume of $1 \mathrm{ml}$ of the appropriate dilution of imipenem was added to $19 \mathrm{ml}$ Muller Hinton agar, cooled to $55^{\circ} \mathrm{C}$ and subsequent to mixing thoroughly, and the mixture was poured onto Petri dishes. The culture grown overnight was collected and the turbidity was matched to McFarland's standard 0.5 (23). A sample of $2 \mu \mathrm{l}$ culture was delivered onto a Petri dish, which was divided into quadrants, and the plate was incubated for $18-24 \mathrm{~h}$ at $37^{\circ} \mathrm{C}$. Following incubation, the highest dilutions exhibiting no visible growth were considered as the MIC of the particular strain (CLSI 2012 guidelines) (23).

Detection of MBLs. Phenotypic detection of MBLs was conducted by the zone enhancement method using ceftazidime discs (Oxoid Ltd) with EDTA (22). Muller Hinton agar plates were seeded with the test organism matched to $0.5 \mathrm{McF}$ arland's

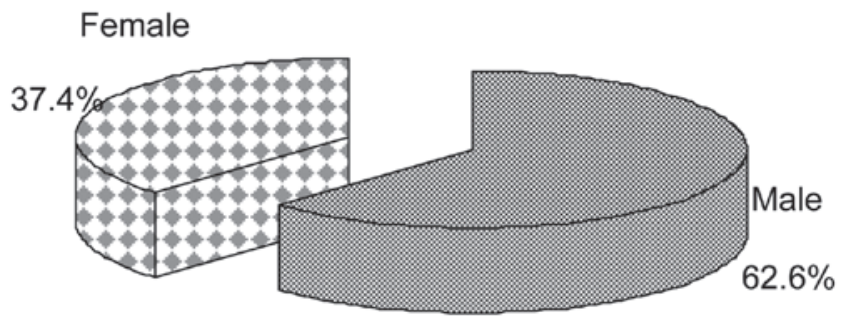

Figure 1. Gender distribution among the cystic fibrosis patients.

standard, according to the CLSI 2012 guidelines (23). A $0.5 \mathrm{M}$ EDTA solution was prepared with $186.1 \mathrm{~g}$ disodium EDTA dissolved in $1.0 \mathrm{ml}$ distilled water at $\mathrm{pH} 8.0$ using $\mathrm{NaOH}$. Subsequent to sterilization by autoclaving, EDTA solution was added to 750- $\mu$ g ceftazidime discs. The discs impregnated with EDTA were dried in the incubator and stored in airtight vials at $-20^{\circ} \mathrm{C}$. The ceftazidime $(30 \mu \mathrm{g})$ discs and ceftazidime-EDTA discs $(750 \mu \mathrm{g})$ were placed upon the agar surface and incubated for $16-18 \mathrm{~h}$ at $35^{\circ} \mathrm{C}$. The zone of enhancement surrounding the ceftazidime EDTA disc was considered to be positive for MBL production.

Genotypic characterization of the MBL gene. DNA was extracted from the $P$. aeruginosa isolates by the boiling method (24). Cultures of $P$. aeruginosa were grown overnight in Trypticase soy broth (Difco Laboratories, Inc., Detroit, MI, USA). A sample of $1.5 \mathrm{ml}$ overnight culture was transferred to an Eppendorf tube and centrifuged at $17,310 \mathrm{x} \mathrm{g}$ in an Eppendorf cooling centrifuge for $5 \mathrm{~min}$. Following centrifugation, the supernatant was decanted and the pellet was suspended in $500 \mu \mathrm{l}$ MilliQ water (Millipore Corp., Billerica, MA, USA). The suspension was boiled at $95^{\circ} \mathrm{C}$ for $10 \mathrm{~min}$ and cell debris was removed by centrifugation at $17,310 \mathrm{x} \mathrm{g}$ for $5 \mathrm{~min}$. The supernatant served as a template for amplification. Duplex polymerase chain reaction (PCR) was performed to detect the presence of blaIMP and blaVIM $\beta$-lactamase in a Thermal Cycler 9600 instrument (Applied Biosystems, Norwalk, CT, USA). The reaction was prepared in a final volume of $50 \mu \mathrm{l}$, containing $10 \mathrm{mM}$ Tris- $\mathrm{HCl}$ (pH 8.3), $50 \mathrm{mM} \mathrm{KCl,} 2.5 \mathrm{mM}$ $\mathrm{MgCl}_{2}, 0.25 \mathrm{mM}$ deoxyribonucleotide triphosphate, $0.75 \mathrm{mM}$ deoxyuridine triphosphate (Roche Diagnostics, Quebec, Canada), 0.125 U uracil-DNA glycosylase and 2 U Taq polymerase (Roche Diagnostics). Concentrations of $0.16 \mathrm{mM}$ of each primer were used in PCR .The primer sequences were as follows: IMP-A, forward 5'-GAAGGYGTTTATGTTCATAC-3' and IMP-B, reverse, 5'-GTAMGTTTCAAGAGTGATGC-3' with a product size of 587 bp (33) and VIM2004A, forward 5'-GTTTGGTCGCATATCGCAAC-3' and VIM2004B, reverse 5'-AATGCGCAGCACCAG GATAG-3' with a 382 bp amplicon size (Roche Diagnostics) A $2-\mu 1$ sample served as a template. The following thermocycling conditions were used for amplification: Initial denaturation step at $94^{\circ} \mathrm{C}$ for $5 \mathrm{~min}$, for 30 cycles followed by denaturation at $94^{\circ} \mathrm{C}$ for $1 \mathrm{~min}$, annealing at $54^{\circ} \mathrm{C}$ for $1 \mathrm{~min}$ and primer extension at $72^{\circ} \mathrm{C}$ for $1.5 \mathrm{~min}$. Subsequent to amplification, the amplicons were visualized on $1.5 \%$ agarose gel in TAE buffer [containing 0.04 M Tris-acetate and $0.002 \mathrm{M}$ EDTA ( $\mathrm{pH} 8.5)]$ to detect the presence of bands and the gels were scanned under ultraviolet illumination, visualized and digitized with a Bio-Rad Gel Doc imaging system (Bio-Rad, 
Table I. Number of Pseudomonas aeruginosa imipenem-resistant isolates at different concentrations of MIC analyzed using the agar dilution method for imipenem.

\begin{tabular}{lcccccccccccccc}
\hline $\mathrm{MIC}(\mu \mathrm{g})$ & 512 & 256 & 128 & 64.0 & 3.02 & 16.0 & 8.00 & 4.00 & 2.00 & 1.00 & 0.500 & 0.250 & 0.125 & 0.0600 \\
\hline $\begin{array}{l}\text { Imipenem- } \\
\begin{array}{l}\text { resistant isolates } \\
(\mathrm{n}=159)\end{array}\end{array}$ & 22 & 16 & 6 & 2 & 8 & 2 & 1 & 5 & 2 & 3 & 1 & 1 & 1 & 2 \\
\hline
\end{tabular}

MIC, minimum inhibitory concentration.

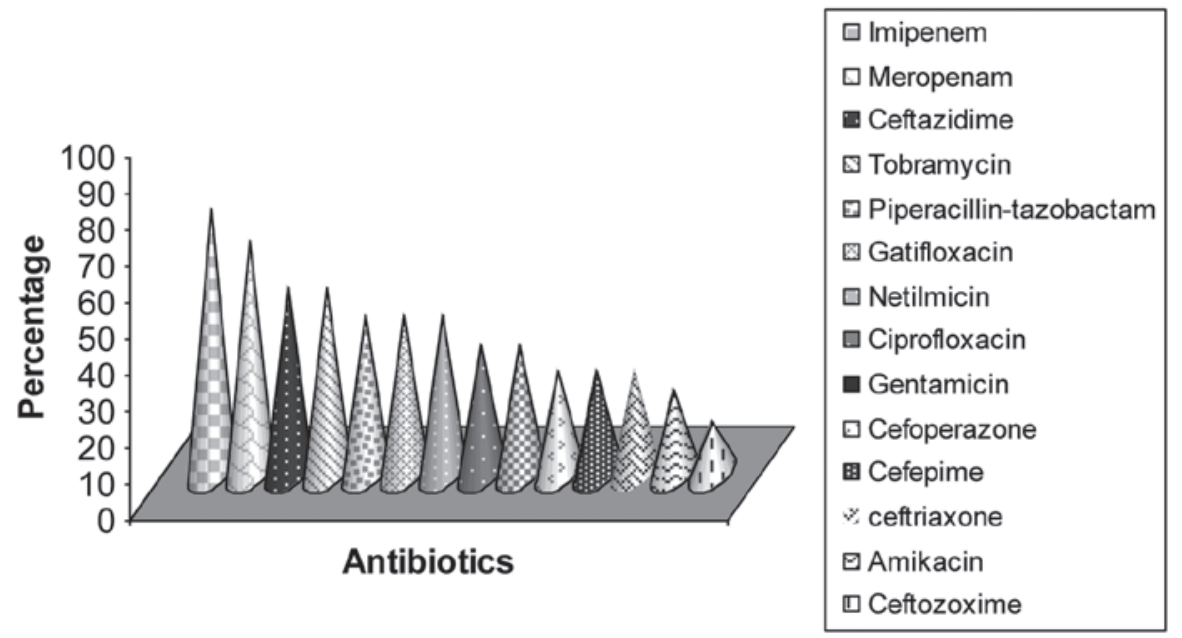

Figure 2. Proportion of Pseudomonas aeruginosa isolates from cystic fibrosis patients resistant to various antibiotics. The present study reported Imipenen resistance as $73.3 \%$. Certain strains were multidrug resistant.

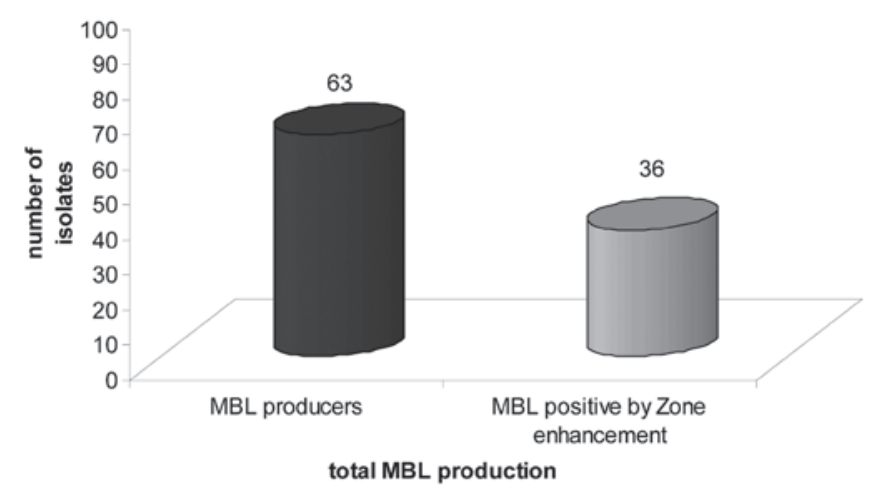

Figure 3. MBL positivity of Pseudomonas aeruginosa samples isolated from cystic fibrosis patients. MBL, metallo- $\beta$-lactamase.

Sydney, Australia). SPSS 11 software (SPSS, Inc., Chicage, IL, USA) was used for statistical analysis of data.

\section{Results}

Patient characteristics. Of the $572 \mathrm{CF}$ patients, 358 (62.6\%) were male and 214 (37.4\%) were female (Fig. 1). P. aeruginosa infection among the CF patients was more prevalent in the $0-5$ year-old group compared with the $>15$ year-old group. Among the 572 patients, 298 (52.1\%) were hospitalized and were aged $<12$ years and found to be culture negative for P. aeruginosa. Out of 572 patients recruited, 217 (37.9\%) were infected with $P$. aeruginosa. No significant correlation was observed between Pseudomonas infection and CF.

Resistance pattern. Of the 217 P. aeruginosa isolates, $159(73.3 \%)$ were resistant to imipenem and 141 (64.9\%) to meropenem. Ceftazidime and tobramycin resistance was detected in 112 (51.6\%) respective samples, and 96 (44.2\%) isolates were resistant to piperacillin-tazobactam, gatifloxacin and netilmicin, respectively. A total of 78 (35.9\%) colonies were resistant to ciprofloxacin and gentamicin, and $62(28.6 \%)$ were resistant to cefoperazone, cefepime and ceftriaxone. The least resistance was observed for amikacin, with 51 (23.5\%) resistant isolates, followed by ceftizoxime with 32 (14.7\%) resistant samples (Fig. 2).

MIC. The MIC for the antibiotics was determined by the agar dilution method according to the CLSI 2012 guidelines (23). Among the 159 imipenem-resistant isolates examined, 72 exhibited a four-fold reduction in MIC values (Table I).

Detection of MBLs. Of the 112 isolates resistant to ceftazidime, $63(56.25 \%)$ were found to be positive for MBL production. A total of $36(57.1 \%)$ showed enhancement of the zone surrounding the ceftazidime-EDTA discs (Fig. 3). One notable feature among the $63 \mathrm{MBL}$-producing $P$. aeruginosa samples was that all strains were found to be resistant to meropenem and ceftazidime. Out of the $63 \mathrm{MBL}$-producing isolates, $38(60.3 \%)$ isolates were from male patients and 


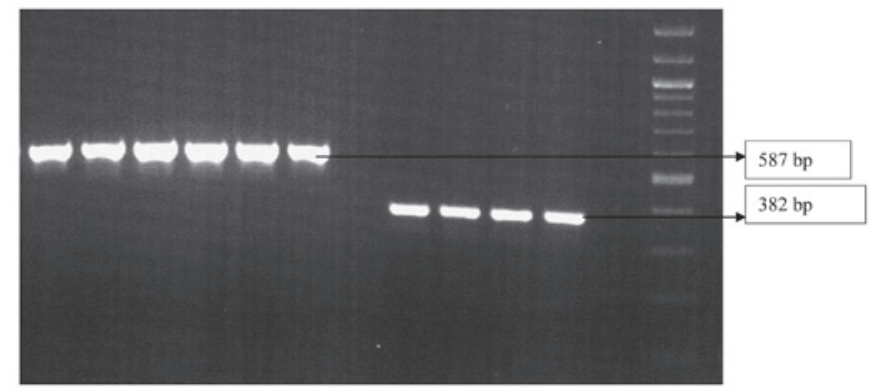

Figure 4. Gel image of DNA extracted from Pseudomonas aeruginosa isolates from cystic fibrosis patients, amplified using polymerase chain reaction. Lanes 1-6 reveal the presence of a blaIMP gene of amplicon size $587 \mathrm{bp}$. Lane 7 is a negative control, with no amplicon. Lanes 8-11 reveal the presence of a blaVIM gene of $382 \mathrm{bp}$ length. Lane 12, $100 \mathrm{bp}$ ladder.

$25(39.7 \%)$ were from female patients; no statistical significance $(\mathrm{P}<0.05)$ was identified between gender and $\mathrm{MBL}$ production. However, statistical significance $(\mathrm{P}<0.05)$ was detected in the association between ceftazidime resistance and MBL production in the isolates.

Genotypic detection of $M B L$ genes. Primers were designed to detect the presence of the $\beta$-lactamase genes blaVIM and blaIMP. Of the $217 P$. aeruginosa strains screened, only 63 isolates were found to produce MBL. These 63 strains were then analyzed by PCR. Out of 63 isolates, 53 (84.1\%) exhibited the presence of blaVIM genes and 48 (76.1\%) exhibited the presence of blaIMP genes (Fig. 4).

\section{Discussion}

Knowledge of the susceptibility of $P$. aeruginosa to antimicrobial agents is urgently required, since understanding of the pattern of antibiotic resistance may aid in treatment of this infection, particularly in CF patients. The prevalence of resistant strains among $\mathrm{CF}$ patients may be elucidated by testing these antibiotics on isolates collected from patients (25). Resistance to carbapenem is of clinical concern $(26,27)$. $P$. aeruginosa is an opportunistic multidrug-resistant pathogen, which is an increasing problem worldwide $(28,29)$. Kulczycki et al (2) revealed a $76.6 \%$ prevalence rate, which is high compared with that of the present study (37.9\%). Various studies of $P$. aeruginosa infection worldwide have observed percentages of resistance to imipenem and meropenem of $4-70 \%$ (5). In the present study, $73.3 \%$ isolates were resistant to imipenem and $64.9 \%$ to meropenem. These are higher values than usual, which reveals that there is increasing resistance of $P$. aeruginosa towards antimicrobial drugs. MBL expression among $P$. aeruginosa samples was found to be $10-65 \%$ across the country from varying clinical samples (15). In the present study, $56.25 \%$ P. aeruginosa isolates produced MBL, which was a lower percentage than that previously identified in a study group of severe acute respiratory infection, defined by the World Health Organisation as an acute respiratory illness of recent onset (within 7 days) manifested by fever $\left(\geq 38^{\circ} \mathrm{C}\right)$, cough and dypnea requiring overnight hospitalization) (30) and a study by Behara et al (31), which observed that $62.5 \%$ P. aeruginosa isolates produced MBL. The higher rate of MBL production among $P$. aeruginosa suggested that carbapenem resistance in $P$. aeruginosa is mediated by $\mathrm{MBL}$ production. Higher morbidity and mortality are associated with $P$. aeruginosa producing MBL (32). In the present study, $36(57.1 \%)$ isolates showed enhancement of the zone surrounding the ceftazidime-EDTA disc, which is lower than the percentage identified by Hemlatha et al (33) (87.5\%), who also observed a lower percentage of isolates producing MBL.

In the present study, the majority of $\mathrm{CF}$ patients recruited were male (62.6\%). Among the 217 isolates of $P$. aeruginosa examined, the antibiogram analysis revealed high resistance to ceftazidime (51.6\%), which was marginally less compared with that reported by Mayank et al (34), who had detected ceftazidime resistance in $63 \%$ of isolates. In other studies by Obritsch et al (35) and Arya et al (36), 55.4\% ceftazidime resistance was observed, which is concordant with the present study. The present study also identified higher resistance to other antibiotics, including tobramycin (51.6\%), piperacillin-tazobactam, gatifloxacin, netilmicin (44.2\%), ciprofloxacin and gentamicin (35.9\%). However, resistance to cefoperazone and cefepime was detected in $28.9 \%$ of samples, which is similar to other studies demonstrating reduced susceptibility to commonly used antibiotics $(3,4,11,12)$.

The 159 isolates resistant to imipenem were examined for MIC by agar dilution. A significant four-fold reduction was observed in the MIC of $72(45.3 \%)$ of these samples. In total, MIC ranges of 0.06- to 512-fold were observed, which is in concordance with previous studies by Migliavacca et al (37) in 2002, Hemlatha et al (33) in 2005, Aggarwal et al (38) in 2008 and Jakumar et al (39) in 2007.

It is important to confirm the presence of the blaVIM and blaIMP $\beta$-lactamase genes by PCR. In the present study, out of the 217 P. aeruginosa strains, the 63 isolates positive for MBL production were selected for PCR analysis. Of the 63 isolates, $53(84.1 \%)$ exhibited the presence of blaVIM genes and 48 (76.1\%) exhibited the presence of blaIMP genes, which corresponds with studies by Mayank et al (34) and Sader et al (40).

In conclusion, the present study emphasizes the requirement for clinical microbiology laboratories to analyze MBL production in carbapenem-resistant $P$. aeruginosa strains. As an increase in multi-drug resistance has been identified among Gram-negative bacteria, an uncontrolled increase in MBL production may result in therapeutic complications, which may in turn raise mortality and morbidity. Early and accurate detection of MBLs may control the spread of MDR pathogens in the future. The use of molecular techniques aids in MBL detection in regional laboratories, to provide the appropriate diagnosis and identification of outbreaks by MBL-producing MDR pathogens, particularly in cystic fibrosis patients. Thus, regular surveillance of MBL-producing P. aeruginosa, along with judicious use of antibiotics, may prevent the spread of drug resistance.

\section{Acknowledgements}

Financial support was provided by the Scientific and technological project of Henan Province (no. 132102310244) and the Henan University of Traditional Chinese Medicine Graduate Innovation Fund Project (no. 201210). 


\section{References}

1. Friend PA: Pulmonary infection in cystic fibrosis. J Infect 13 55-72, 1986.

2. Kulczycki LL, Murphy TM and Bellanti JA: Pseudomonas colonization in cystic fibrosis. A study of 160 patients. JAMA 240 : 30-34, 1978.

3. May JR, Herrick NC and Thompson D: Bacterial infection in cystic fibrosis. Arch Dis Child 47: 908-913, 1972.

4. Mearns MB, Hunt GH and Rushworth R: Bacterial flora of respiratory tract in patients with cystic fibrosis, 1950-1971. Arch Dis Child 47: 902-907, 1972.

5. Thomassen MJ, Demko CA, Boxerbaum B, et al: Multiple isolates of Pseudomonas aeruginosa with differing antimicrobia susceptibility patterns from patients with cystic fibrosis. J Infect Dis 140: 873-880, 1979.

6. Arancibia F, Bauer TT, Ewig S, et al: Community-acquired pneumonia due to gram-negative bacteria and Pseudomonas aeruginosa: incidence, risk, and prognosis. Arch Intern Med 162: 1849-1858, 2002.

7. West SE, Zeng L, Lee BL, et al: Respiratory infections with Pseudomonas aeruginosa in children with cystic fibrosis: early detection by serology and assessment of risk factors. JAMA 287: 2958-2967, 2002.

8. Spilker T, Coenye T, Vandamme P, and LiPuma JJ: PCR-based assay for differentiation of Pseudomonas aeruginosa from other Pseudomonas species recovered from cystic fibrosis patients. J Clin Microbiol 42: 2074-2079, 2004

9. Gillespie SH and Hawkey PM: Principles and Practice of Clinical Bacteriology. 2nd edition. Wiley, Chichester, UK. 2006.

10. Lyczak JB, Cannon CL and Pier GB: Lung infections associated with cystic fibrosis. Clin Microbiol Rev 15: 194-222, 2002.

11. Nikaido H: Multidrug efflux pumps of gram-negative bacteria J Bacteriol 178: 5853-5859, 1996

12. Sonnesyn SW and Gerding DN: Antimicrobials for the treatment of respiratory infections. In: Respiratory Infections: A Scientific Basis for Management. Niederman MS, Sarosi GA and Glassroth J (eds). Saunders, Philadelphia, pp511-537, 1994.

13. Lee K, Lim YS, Yong D, et al: Evaluation of the Hodge test and the imipenem-EDTA double-disk synergy test for differentiating metallo-beta-lactamase-producing isolates of Pseudomonas spp. and Acinetobacter spp. J Clin Microbiol 41: 4623-4629, 2003.

14. Varaiya A, Kulkarni N, Kulkarni M, et al: Incidence of metallo beta lactamase producing Pseudomonas aeruginosa in ICU patients. Indian J Med Res 127: 398-402, 2008.

15. Oh EJ,Lee S, Park YJ, et al: Prevalence of metallo-beta-lactamase among Pseudomonas aeruginosa and Acinetobacter baumanni in a Korean university hospital and comparison of screening methods for detecting metallo-beta-lactamase. J Microbiol Methods 54: 411-418, 2003

16. Pitout JD, Gregson DB, Poirel L, et al: Detection of Pseudomonas aeruginosa producing metallo-beta-lactamases in a large centralized laboratory. J Clin Microbiol 43: 3129-3135, 2005.

17. Gladstone P, Rajendran P and Brahmadathan KN: Incidence of carbapenem resistant nonfermenting gram negative bacilli from patients with respiratory infections in the intensive care units. Indian J Med Microbiol 23: 189-191, 2005.

18. Cornaglia G, Mazzariol A, Lauretti L, et al: Hospital outbreak of carbapenem-resistant Pseudomonas aeruginosa producing VIM-1, a novel transferable metallo-beta-lactamase. Clin Infect Dis 31: $1119-1125,2000$.

19. Crespo MP, Woodford N, Sinclair A, et al: Outbreak of carbapenem-resistant Pseudomonas aeruginosa producing VIM-8, a novel metallo-beta-lactamase, in a tertiary care center in Cali, Colombia. J Clin Microbiol 42: 5094-5101, 2004.

20. Pournaras S, Maniati M, Petinaki E, et al: Hospital outbreak of multiple clones of Pseudomonas aeruginosa carrying the unrelated metallo-beta-lactamase gene variants $b l a_{\mathrm{VIM}-2}$ and

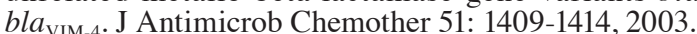

21. Tsakris AS, Pournaras S, Woodford M, et al: Outbreak of infections caused by Pseudomonas aeruginosa producing VIM-1 carbapenemase in Greece. J Clin Microbiol 38: 1290-1292, 2000.
22. Yong D, Lee K, Yum JH, et al: Imipenem-EDTA disk method for differentiation of metallo-beta-lactamase-producing clinical isolates of Pseudomonas spp. and Acinetobacter spp. J Clin Microbiol 40: 3798-3801, 2002

23. Clinical and Laboratory Standards Institute (CLSI): Performance standards for antimicrobial susceptibility testing. 11 th informational supplement. CLSI, Wayne, PA, USA, M100-S23, 2013.

24. Hall BG and Barlow M: Revised Ambler classification of $\beta$-lactamases. J Antimicrob Chemother 55: 1050-1051, 2005.

25. Pitt TL, Sparrow M, Warner M and Stefanidou M: Survey of resistance of Pseudomonas aeruginosa from UK patients with cystic fibrosis to six commonly prescribed antimicrobial agents. Thorax 58: 794-796, 2003.

26. Cheng K, Smyth RL, Govan JR, et al: Spread of beta-lactam-resistant Pseudomonas aeruginosa in a cystic fibrosis clinic. Lancet 348: 639-642, 1996.

27. Gales AC, Jones RN, Turnidge J, et al: Characterization of Pseudomonas aeruginosa isolates: occurrence rates, antimicrobial susceptibility patterns, and molecular typing in the global SENTRY antimicrobial surveillance program, 1997-1999. Clin Infect Dis 32 Suppl 2: S146-S155, 2001.

28. Neuhauser MM, Weinstein RA, Rydman R, et al: Antibiotic resistance among gram-negative bacilli in US intensive care units: implications for fluoroquinolone use. JAMA 289: 885-888, 2003.

29. Cardoso O, Alves AF and Leitão R: Metallo-beta-lactamase VIM-2 in Pseudomonas aeruginosa isolates from a cystic fibrosis patient. Int J Antimicrob Agents 31: 375-379, 2008

30. Manoharan A, Chatterjee S and Mathai D; SARI Study Group: Detection and characterization of metallo beta lactamases producing Pseudomonas aeruginosa. Indian J Med Microbiol 28: 241-244, 2010.

31. Behera B, Mathur P, Das A, et al: An evaluation of four different phenotypic techniques for detection of metallo-beta-lactamase producing Pseudomonas aeruginosa. Indian J Med Microbiol 26: 233-237, 2008.

32. Hemlatha V, Sekar U and Kamat V: Detection of metallo betalactamase producing Pseudomonas aeruginosa in hospitalized patients. Indian J Med Res 122: 148-152, 2005

33. Hemlatha V, Sekar U and Kamat V: Detection of metallo betalactamase producing Pseudomonas aeruginosa in hospitalized patients. Indian J Med Res 122: 148-152, 2005.

34. Mayank D, Anshuman M, Singh RK, et al: Nosocomial cross-transmission of Pseudomonas aeruginosa between patients in a tertiary intensive care unit. Indian J Pathol Microbiol 52: 509-513, 2009.

35. Obritsch MD, Fish DN, MacLaren R and Jung R: National surveillance of antimicrobial resistance in Pseudomonas aeruginosa isolates obtained from intensive care unit patients from 1993 to 2002. Antimicrob Agents Chemother 48: 4606-4610, 2004.

36. Arya M, Arya PK, Biswas D and Prasad R: Antimicrobial susceptibility pattern of bacterial isolates from post-operative wound infections. Indian J Pathol Microbiol 48: 266-269, 2005.

37. Migliavacca R, Docquier JD, Mugnaioli C, et al: Simple microdilution test for detection of metallo- $\beta$-lactamase production in Pseudomonas aeruginosa. J Clin Microbiol 40: 4388-4390, 2002.

38. Aggarwal R, Chaudhary $U$ and Bala K: Detection of extended-spectrum beta lactamase in Pseudomonas aeruginosa. Indian J Pathol Microbiol 51: 222-224, 2008.

39. Jayakumar S and Appalaraju B: Prevalence of multi and pan drug resistant Pseudomonas aeruginosa with respect to ESBL and MBL in a tertiary care hospital. Indian J Pathol Microbiol 50: 922-925, 2007.

40. Sader HS, Reis AO, Silbert S and Gales AC: IMPs, VIMs and SPMs: the diversity of metallo- $\beta$-lactamases produced by carbapenem-resistant Pseudomonas aeruginosa in a Brazilian hospital. Clin Microbiol Infect 11: 73-76, 2005. 